\title{
Generalized Brillinger-Like Transforms
}

\author{
Anatoli Torokhti and Pablo Soto-Quiros
}

\begin{abstract}
We propose novel transforms of stochastic vectors, called the generalized Brillinger transforms (GBT1 and GBT2), which are generalizations of the Brillinger transform (BT). The GBT1 extends the BT to the cases when the covariance matrix and the weighting matrix are singular, and moreover, the weighting matrix is not necessarily symmetric. We show that the GBT1 may computationally be preferable over another related optimal technique, the generic Karhunen-Loève transform (GKLT). The GBT2 generalizes the GBT1 to provide, under the condition we impose, better associated accuracy than that of the GBT1. It is achieved because of the increase in a number of parameters to optimize compared to that in the GBT1.
\end{abstract}

Index Terms-Brillinger transform (BT), data compression, filtering.

\section{INTRODUCTION}

Motivation: Recently, the transform of stochastic vectors proposed by Brillinger [1, pp. 368-69, 457] has received considerable attention in signal processing problems (see, e.g., [2]-[7]) as an effective tool for data compression and filtering. The Brillinger transform (BT) $F$ is represented in terms of two matrices, $F=A C$, where $C$ is designated for compression and $A$ for decompression (they are called "compressor" and "decompressor," respectively). We wish to extend the BT to more efficient transforms.

Contribution and novelty: The contribution of this work is threefold. First, in Section II, we extend the BT to a generalized first-order BT (GBT1) which is applicable to the cases when the covariance matrix and the weighting matrix are singular, and moreover, the weighting matrix is not necessarily symmetric. The proposed GBT1 is given in terms of pseudoinverse matrices and therefore, it always exists, i.e., it is applicable to cases where the BT is not. This is because BT performs only under the assumption that the covariance matrix and weighting matrix are invertible, and that the weighting matrix is symmetric. These conditions might be restrictive [8] in problems where the BT is applied. We note that the extension of the BT to the GBT1 is not straightforward and requires a new justification based on Facts 1-3 in Section II-A.

Second, although the GBT1 provides the best decompression accuracy, for the same compression ratio, among all transforms of the form $F=A C$ it may happen that the accuracy

Manuscript received February 08, 2016; revised April 12, 2016; accepted April 18, 2016. Date of publication April 20, 2016; date of current version May 05,2016 . The associate editor coordinating the review of this manuscript and approving it for publication was Prof. Marco Lops.

A. Torokhti is with the Centre for Industrial and Applied Mathematics, University of South Australia, Adelaide, S.A. 5095, Australia (e-mail: anatoli. torokhti@unisa.edu.au).

P. Soto-Quiros is with the Centre for Industrial and Applied Mathematics, University of South Australia, Adelaide, S.A. 5095, Australia, and also with the Instituto Tecnologico de Costa Rica, Cartago 30101, Costa Rica (e-mail: juan. soto-quiros@mymail.unisa.edu.au).

Color versions of one or more of the figures in this letter are available online at http://ieeexplore.ieee.org.

Digital Object Identifier 10.1109/LSP.2016.2556714 is still not satisfactory. In Section III, we propose and justify a new transform called the generalized second-order BT (GBT2) which provides, under the condition we impose, better associated accuracy than that of the GBT1.

Third, we show that the GBT1 requires a lower computational load than other related technique, the generic KarhunenLoève transform (GKLT) [8]-[10]. This issue is elaborated in Section III. Such an analysis provides a better understanding of the two well-known data compression techniques.

\section{A. Truncated SVD}

Let the SVD of matrix $M \in \mathbb{R}^{m \times n}$ be given by $M=$ $U_{M} \Sigma_{M} V_{M}^{T}$, where $U_{M}=\left[\begin{array}{llll}u_{1} & u_{2} & \ldots & u_{m}\end{array}\right] \in \mathbb{R}^{m \times m}, V_{M}=$ $\left[\begin{array}{llll}v_{1} & v_{2} & \ldots & v_{n}\end{array}\right] \in \mathbb{R}^{n \times n}$ are unitary matrices, and $\Sigma_{M}=$ $\operatorname{diag}\left(\sigma_{1}(M), \ldots, \sigma_{\min (m, n)}(M)\right) \in \mathbb{R}^{m \times n}$ is a generalized diagonal matrix, with the singular values $\sigma_{1}(M) \geq \sigma_{2}(M) \geq$ $\cdots \geq 0$ on the main diagonal. Let

$P_{M, L}=\sum_{k=1}^{\operatorname{rank}(M)} u_{k} u_{k}^{T} \in \mathbb{R}^{m \times m}, \quad P_{M, R}=\sum_{j=1}^{\operatorname{rank}(M)} v_{j} v_{j}^{T} \in \mathbb{R}^{n \times n}$

be the orthogonal projections on the range of $M$ and $M^{T}$, respectively, and let

$$
[M]_{k}=\sum_{i=1}^{k} \sigma_{i}(M) u_{i} v_{i}^{T} \in \mathbb{R}^{m \times n}
$$

for $k=1, \ldots$, rank $(M)$, be the truncated SVD of $M$. For $k>\operatorname{rank}(M)$, we define $[M]_{k}=M\left(=M_{\text {rank }(M)}\right)$. For $1 \leq$ $k<\operatorname{rank}(M)$, the matrix $M_{k}$ is uniquely defined if and only if $\sigma_{k}(M)>\sigma_{k+1}(M)$.

\section{B. Special Notation}

Let us write $(\Omega, \Sigma, \mu)$ for a probability space. ${ }^{1}$ We denote by $\mathbf{y}=\left(\mathbf{y}_{1}, \ldots, \mathbf{y}_{m}\right)^{T} \in L^{2}\left(\Omega, \mathbb{R}^{m}\right)$ the signal of interest $t^{2}(\mathrm{a}$ source signal to be estimated) and by $\mathbf{x}=\left(\mathbf{x}_{1}, \ldots, \mathbf{x}_{n}\right)^{T} \in$ $L^{2}\left(\Omega, \mathbb{R}^{n}\right)$ the observed signal where $\mathbf{y}_{j}, \mathbf{x}_{k} \in L^{2}(\Omega, \mathbb{R})$. Let us write $\mathbb{E}\left[\|\mathbf{x}\|_{2}^{2}\right]=\int_{\Omega}\|\mathbf{x}(\omega)\|_{2}^{2} d \mu(\omega)$, where $\|\mathbf{x}(\omega)\|_{2}$ is the Euclidean norm of $\mathbf{x}(\omega) \in \mathbb{R}^{m}$. We assume that means $E[\mathbf{y}]$ and $E[\mathbf{x}]$ are known. Therefore, without loss of generality, we will assume henceforth that $\mathbf{y}$ and $\mathbf{x}$ have zero means. Then, $E_{\mathbf{y x}}=E\left[\mathbf{y} \mathbf{x}^{T}\right]=\left\{e_{i j}\right\}_{i, j=1}^{m, n} \in \mathbb{R}^{m \times n}$, where $e_{i j}=\int_{\Omega} \mathbf{y}_{i}(\omega) \mathbf{x}_{j}(\omega) d \mu(\omega)$.

Furthermore, $M^{\dagger}$ and $M^{1 / 2}$ denote the Moore-Penrose pseudoinverse matrix and a matrix square root, respectively, for matrix $M$. For the covariance matrix $E_{\mathbf{x x}}$, we denote $E_{\mathbf{x x}}^{1 / 2 \dagger}:=$ $\left(E_{\mathbf{x x}}^{1 / 2}\right)^{\dagger} \cdot E_{\mathbf{x x}}^{1 / 2 \dagger}$ is unique since $E_{\mathbf{x x}}$ is positive semidefinite.

${ }^{1} \Omega=\{\omega\}$ is the set of outcomes, $\Sigma$ a $\sigma$-field of measurable subsets of $\Omega$, $\mu: \Sigma[0,1]$ an associated probability measure on $\Sigma$ with $\mu(\Omega)=1$.

${ }^{2} L^{2}\left(\Omega, \mathbb{R}^{m}\right)$ is the space of square-integrable functions defined on $\Omega$ with values in $\mathbb{R}^{m}$, i.e., such that $\int_{\Omega}\|\mathbf{x}(\omega)\|_{2}^{2} d \mu(\omega)<\infty$. 


\section{Review of the $B T$}

Let $k \leq \min \{m, n\}, \Gamma \in \mathbb{R}^{m \times m}$ be a symmetric invertible weighting matrix, $A \in \mathbb{R}^{m \times k}$ and $C \in \mathbb{R}^{k \times n}$ be matrices of a decompressor and compressor, respectively. The BT of $\mathbf{x}$ is represented by matrices $A$ and $C$ that solve

$$
\min _{A, C} \mathbb{E}\left[\|\Gamma(\mathbf{y}-A C \mathbf{x})\|_{2}^{2}\right]
$$

Weighting matrix $\Gamma$ is used, based on a priori information, to place a greater importance on some particular entries of the observed data (in this regard, see, e.g., [11]-[13]). ${ }^{3}$ Denote $T=\Gamma E_{\mathbf{y x}} E_{\mathbf{x x}}^{-1} E_{\mathbf{x y}} \Gamma$ and suppose that the SVD of $T$ is given by $T=U_{T} \Sigma_{T} U_{T}^{T}$. Under the assumption that $E_{\mathbf{x x}}$ is invertible, the BT in [1] is given by

$$
C=U_{T, k}^{T} \Gamma E_{\mathbf{y x}} E_{\mathbf{x x}}^{-1} \quad \text { and } \quad A=\Gamma^{-1} U_{T, k}
$$

where $U_{T, k}$ is formed by the first $k$ columns of $U_{T}$.

\section{Generalized First-Order BT (GBT1)}

\section{A. Derivation and Justification of GBT1}

Here, we consider the generalized BT (GBT1) which is an extension of the BT to the case in which $E_{\mathbf{x x}}$ and $\Gamma$ are not invertible, and moreover, $\Gamma$ is not necessarily symmetric.

To this end, denote $T_{x}=\Gamma E_{\mathbf{y x}} E_{\mathbf{x x}}^{\dagger} E_{\mathbf{x y}} \Gamma^{T}, U_{T_{x}} \Sigma_{T_{x}} U_{T_{x}}^{T}=$ $T_{x}$ is the SVD of $T_{x}, M_{L}=I+\left(I-P_{\Gamma, R}\right) S$ and $M_{R}=$ $I+R\left(I-P_{E_{\mathbf{x}}^{1 / 2}, L}\right)$, where $S$ and $R$ are arbitrary matrices. Furthermore, $\|\cdot\|$ denotes the Frobenius matrix norm.

Theorem 1: The GBT1 is represented by matrices $A_{\mathrm{GB}} \in$ $\mathbb{R}^{m \times k}$ and $C_{\mathrm{GB}} \in \mathbb{R}^{k \times n}$ such that

$$
A_{\mathrm{GB}}=M_{L} \Gamma^{\dagger} U_{T_{x}, k} \quad \text { and } \quad C_{\mathrm{GB}}=U_{T_{x}, k}^{T} \Gamma E_{\mathbf{y x}} E_{\mathbf{x x}}^{\dagger} M_{R} .
$$

The minimal norm GBT1 is given by $A_{\mathrm{GB}}=\Gamma^{\dagger} U_{T_{x}, k}$ and $C_{\mathrm{GB}}=U_{T_{x}, k}^{T} \Gamma E_{\mathbf{y x}} E_{\mathbf{x x}}^{\dagger}$. For the fixed compression ratio $c=$ $\frac{k}{\min \{m, n\}}$, the associated error is given by

$$
\begin{aligned}
\varepsilon_{\mathrm{GBT} 1} & =\min _{A, C} \mathbb{E}\left[\|\Gamma(\mathbf{y}-A C \mathbf{x})\|_{2}^{2}\right] \\
& =\left\|\left(\Gamma E_{\mathbf{y y}} \Gamma^{T}\right)^{1 / 2}\right\|^{2}-\alpha_{1}
\end{aligned}
$$

where $\alpha_{1}=\sum_{j=1}^{r_{x}} \sigma_{j}\left(T_{x}\right), r_{x}=k$ if $k \leq \operatorname{rank}\left(T_{x}\right)$ and $r_{x}=$ $\operatorname{rank}\left(T_{x}\right)$ if $k>\operatorname{rank}\left(T_{x}\right)$.

Remark 1: If $E_{\mathbf{x x}}^{\dagger}=E_{\mathbf{x x}}^{-1}$, and matrix $\Gamma$ is symmetric and invertible then (5) represents the error associated with the BT.

The proof of Theorem 1 is based on the following facts.

Fact 1: Let $U_{M} \Sigma_{M} V_{M}^{T}=M$ be the SVD of $M \in \mathbb{R}^{m \times n}$, $\operatorname{rank}(M)=r, k<r, U_{M, k}=\left[\begin{array}{llll}u_{1} & u_{2} & \ldots & u_{k}\end{array}\right]$. Then,

$$
[M]_{k}=U_{M, k} U_{M, k}^{T} M .
$$

For $k \geq r,[M]_{k}=[M]_{r}=M$.

Proof: For convenience, we denote $U_{k}=U_{M, k}, \Sigma_{k}=$ $\operatorname{diag}\left(\sigma_{1}, \ldots, \sigma_{k}\right), \sigma_{j}=\sigma_{j}(M), j=1, \ldots, k$, and $V_{k}=\left[v_{1}\right.$, $\left.\ldots, v_{k}\right]$. Furthermore, let $\mathbb{O}_{k, r}$ be $k \times r$ zero matrix and let

$$
\begin{aligned}
U_{r-k} & =\left[u_{k+1}, \ldots, u_{r}\right], \quad V_{r-k}=\left[v_{k+1}, \ldots, v_{r}\right] \\
\Sigma_{r-k}: & =\operatorname{diag}\left(\sigma_{k+1}, \ldots, \sigma_{r}\right) \quad \text { and } \quad \mathbb{O}_{k, r-k} \in \mathbb{R}^{k \times(r-k)} .
\end{aligned}
$$

\footnotetext{
${ }^{3}$ It is customary to choose small weights where the errors associated with particular entries of observed data are expected to be large, and vice versa.
}

Then, for $I_{k} \in \mathbb{R}^{k \times k}$, (1) implies

$$
\begin{aligned}
{[M]_{k} } & =U_{k}\left[I_{k} \mathbb{O}_{k, r-k}\right]\left[\begin{array}{cc}
\Sigma_{k} & \mathbb{O}_{k, r-k} \\
\mathbb{O}_{k, r-k}^{T} & \Sigma_{r-k}
\end{array}\right] V_{r}^{T} \\
& =U_{k} U_{k}^{T}\left[U_{k} U_{r-k}\right] \Sigma_{r} V_{r}^{T}=U_{k} U_{k}^{T} M .
\end{aligned}
$$

We write $\mathcal{N}(M)$ for the null space of matrix $M$. Fact 2: Let $P \in \mathbb{R}^{n \times m}$ and $Q \in \mathbb{R}^{n \times m}$. Then,

$$
\mathcal{N}\left(P^{T}\right) \subseteq \mathcal{N}\left(Q^{T}\right) \Rightarrow P P^{\dagger} Q=Q .
$$

Proof: By Lemma 23 in [8, p. 167]

$$
\mathcal{N}\left(P^{T}\right) \subseteq \mathcal{N}\left(Q^{T}\right) \Rightarrow Q^{T}\left(P^{\dagger}\right)^{T} P^{T}=Q^{T} .
$$

Then, (7) follows.

Fact 3: The following equality holds:

$$
\Gamma \Gamma^{\dagger}\left[\Gamma E_{\mathbf{y x}} E_{\mathbf{x x}}^{1 / 2}\right]_{k}=\left[\Gamma E_{\mathbf{y x}} E_{\mathbf{x x}}^{1 / 2^{\dagger}}\right]_{k} .
$$

Proof: We have $\mathcal{N}\left(\Gamma^{T}\right) \subseteq \mathcal{N}\left(E_{\mathbf{x x}}^{1 / 2^{\dagger}} E_{\mathbf{y x}}^{T} \Gamma^{T}\right)$ and $\mathcal{N}\left(E_{\mathbf{x x}}^{1 / 2^{\dagger}} E_{\mathbf{y x}}^{T} \Gamma^{T}\right) \subseteq \mathcal{N}\left(\left[E_{\mathbf{x x}}^{1 / 2^{\dagger}} E_{\mathbf{y x}}^{T} \Gamma^{T}\right]_{k}\right)$. Thus, $\mathcal{N}\left(\Gamma^{T}\right) \subseteq$ $\mathcal{N}\left(\left[E_{\mathbf{x x}}^{1 / 2^{\dagger}} E_{\mathbf{y x}}^{T} \Gamma^{T}\right]_{k}\right)$. Then, (7) implies (8).

Proof of Theorem 1: Let us denote $\mathbf{w}=\Gamma \mathbf{y}$. Recall that $\mathbb{E}\left[\|\mathbf{w}\|_{2}^{2}\right]=\int_{\Omega} \operatorname{tr}\left\{\mathbf{w}(\omega) \mathbf{w}(\omega)^{T}\right\} d \mu(\omega)=\operatorname{tr}\left(E_{\mathbf{w w}}\right)$ (since $\|M\|^{2}=\operatorname{tr}\left(M M^{T}\right)$; see, e.g., [8, p. 167]). Then, for $F_{1}=A C$

$$
\begin{aligned}
\mathbb{E}[ & \left.\|\Gamma(\mathbf{y}-A C \mathbf{x})\|_{2}^{2}\right] \\
= & \operatorname{tr}\left\{E_{\mathbf{w} \mathbf{w}}-E_{\mathbf{w} \mathbf{x}} F_{1}^{T} \Gamma^{T}-\Gamma F_{1} E_{\mathbf{x w}}+\Gamma F_{1} E_{\mathbf{x x}} F_{1}^{T} \Gamma^{T}\right\} \\
= & \left\|E_{\mathbf{w} \mathbf{w}}^{1 / 2}\right\|^{2}-\left\|E_{\mathbf{w} \mathbf{x}} E_{\mathbf{x x}}^{1 / 2 \dagger}\right\|^{2} \\
& +\left\|E_{\mathbf{w} \mathbf{x}} E_{\mathbf{x x}}^{1 / 2^{\dagger}}-\Gamma F_{1} E_{\mathbf{x x}}^{1 / 2}\right\|^{2} .
\end{aligned}
$$

The latter is true because $E_{\mathbf{w x}} E_{\mathbf{x x}} E_{\mathbf{x x}}^{\dagger}=E_{\mathbf{w x}}$ (see [8, p. 168]) and $E_{\mathbf{x} \mathbf{x}}^{\dagger} E_{\mathbf{x x}}^{1 / 2}=E_{\mathbf{x x}}^{1 / 2 \dagger}$. Denote by $\mathbb{R}(m, n, k)$ the set of all $m \times n$ matrices of rank at most $k$. It has been shown in [14] and [15] that the solution to problem

$$
\min _{F_{1} \in \mathbb{R}(m, n, k)}\left\|E_{\mathbf{w} \mathbf{x}} E_{\mathbf{x x}}^{1 / 2^{\dagger}}-\Gamma F_{1} E_{\mathbf{x x}}^{1 / 2}\right\|^{2}
$$

is given by

$$
F_{1}=M_{L} \Gamma^{\dagger}\left[\Gamma E_{\mathbf{y x}} E_{\mathbf{x x}}^{1 / 2^{\dagger}}\right]_{k} E_{\mathbf{x x}}^{1 / 2^{\dagger}} M_{R}
$$

(see Remark 2 below), where by Fact 1

$$
\left[\Gamma E_{\mathbf{y x}} E_{\mathbf{x x}}^{1 / 2 \dagger}\right]_{k}=U_{T, k} U_{T, k}^{T} \Gamma E_{\mathbf{y x}} E_{\mathbf{x x}}^{1 / 2^{\dagger}} .
$$

If we denote $F_{1}=F_{\mathrm{GB} 1}=A_{\mathrm{GB}} C_{\mathrm{GB}}$ then (11) and (12) imply (4). Furthermore, if in (9), $F_{1}$ is replaced with $F_{\mathrm{GB} 1}$ then

$$
\begin{aligned}
& \Gamma F_{1} E_{\mathbf{x x}}^{1 / 2} \\
& \quad=\Gamma M_{L} \Gamma^{\dagger}\left[\Gamma E_{\mathbf{y x}} E_{\mathbf{x x}}^{1 / 2 \dagger}\right]_{k} E_{\mathbf{x x}}^{1 / 2 \dagger} M_{R} E_{\mathbf{x x}}^{1 / 2}
\end{aligned}
$$

where $\Gamma M_{L} \Gamma^{\dagger}=\Gamma \Gamma^{\dagger}$ and $E_{\mathbf{x x}}^{1 / 2 \dagger} M_{R} E_{\mathbf{x x}}^{1 / 2}=E_{\mathbf{x x}}^{1 / 2 \dagger} E_{\mathbf{x x}}^{1 / 2}$. Therefore, (13) implies

$$
\Gamma T E_{\mathbf{x x}}^{1 / 2}=\Gamma \Gamma^{\dagger}\left[\Gamma E_{\mathbf{y x}} E_{\mathbf{x x}}^{1 / 2 \dagger}\right]_{k} E_{\mathbf{x x}}^{1 / 2 \dagger} E_{\mathbf{x x}}^{1 / 2}
$$


Here, on the basis of Lemma 42 in [8, p. 311]

$$
\left[\Gamma E_{\mathbf{y x}} E_{\mathbf{x x}}^{1 / 2^{\dagger}}\right]_{k} E_{\mathbf{x x}}^{1 / 2{ }^{\dagger}} E_{\mathbf{x x}}^{1 / 2}=\left[\Gamma E_{\mathbf{y x}} E_{\mathbf{x x}}^{1 / 2^{\dagger}}\right]_{k}
$$

and (8) is also true. Thus,

$$
\begin{aligned}
& \Gamma M_{L} \Gamma^{\dagger}\left[\Gamma E_{\mathbf{y x}} E_{\mathbf{x x}}^{1 / 2^{\dagger}}\right]_{k} E_{\mathbf{x x}}^{1 / 2^{\dagger}} M_{R} E_{\mathbf{x x}}^{1 / 2} \\
& \quad=\left[\Gamma E_{\mathbf{y x}} E_{\mathbf{x x}}^{1 / 2{ }^{\dagger}}\right]_{k} .
\end{aligned}
$$

Then, for $F_{1}=F_{\mathrm{GB} 1}=A_{\mathrm{GB}} C_{\mathrm{GB}}$ and $A_{\mathrm{GB}}, C_{\mathrm{GB}}$ given by (4), the last term in (9) is written as

$$
\begin{aligned}
\left\|\Gamma E_{\mathbf{y x}} E_{\mathbf{x x}}^{1 / 2^{\dagger}}-\Gamma F_{\mathrm{GB} 1} E_{\mathbf{x x}}^{1 / 2}\right\|^{2} \\
\quad=\left\|\Gamma E_{\mathbf{y x}} E_{\mathbf{x x}}^{1 / 2^{\dagger}}-\left[\Gamma E_{\mathbf{y x}} E_{\mathbf{x x}}^{1 / 2^{\dagger}}\right]_{k}\right\|^{2}=\sum_{j=k+1}^{\operatorname{rank}\left(T_{x}\right)} \sigma_{j}\left(T_{x}\right)
\end{aligned}
$$

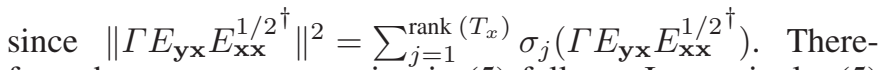
fore, the error representation in (5) follows. Interestingly, (5) does not depend on $S$ and $R$, i.e., any their choice (e.g., $S=$ $\left.\mathbb{O}_{m, m}, R=\mathbb{O}_{n, n}\right)$ results in the same associated error.

Remark 2: In [14] and [15], the proof of (11) is based on $\left\|\Lambda-\Gamma F_{1} G\right\|=\|\widetilde{\Lambda}-Z\|$, where $\Lambda=E_{\mathbf{w x}} E_{\mathbf{x x}}^{1 / 2^{\dagger}}, G=E_{\mathbf{x x}}^{1 / 2}$, $\widetilde{\Lambda}=U_{\Gamma}^{T} \Lambda V_{G}, Z=\Sigma_{\Gamma} \widetilde{F_{1}} \Sigma_{G} \widetilde{F_{1}}=V_{\Gamma}^{T} F_{1} U_{G}$.

Example 1: Let $E_{\mathbf{y x}}, E_{\mathbf{x x}}$ be formed by $6 \times 3$ sample matrices $Y$ and $X=Y+0.1 \Theta$, where $Y$ has uniformly distributed entries and $\Theta$ has normally distributed entries with mean 0 and variance 1 . The entries are chosen randomly. Then, $E_{\mathbf{y x}}$ and $E_{\mathbf{x x}}$ are singular and the BT is not applicable. The GBT1 is applicable and, for $k=2$ and diagonal $\Gamma$ with random entries within $(0,1)$, the associated error is 0.02 .

\section{B. Numerical Load. Comparison With the GKLT}

In a number of applied problems, dimensions $m, n$ of associated covariance matrices are large. For instance, in the DNA array analysis [16], [17], $m=O\left(10^{4}\right)$. In this case, the associated numerical load needed to compute the covariance matrices increases significantly. Therefore, a method which requires a lower associated numerical load is, of course, more preferable. In addition to the advantages of the GBT1 mentioned in Section I, another advantage is that the computational load for the GBT1 is less than that for the GKLT [8]. The GKLT is the optimal technique related to GBT1 and is represented here by (11). The computational schemes for the GBT1 and GKLT are different. In particular, the GBT1 requires to compute $U_{T_{x}, k}$ from the eigendecomposition of the $m \times m$ symmetric matrix $T_{x}$, whereas the GKLT requires to compute the whole SVD of the nonsymmetric $m \times n$ matrix $\Gamma E_{\mathbf{y x}} E_{\mathbf{x x}}^{1 / 2^{\dagger}}$.

Table I provides estimates of the number of flops required to compute $U_{T_{x}, k}$, for the GBT1, and the SVD of $\Gamma E_{\mathbf{y x}} E_{\mathbf{x x}}^{1 / 2^{\dagger}}$, for the GKLT, by the Golub-Reinsch SVD method and the $R$ bidiagonalization method ( $R$-SVD), for $m=n$, as it given in [18, p. 254].

The GBT1 computational load $\left(L_{\mathrm{GBT} 1}\right)$ consists of computation of matrices $E_{\mathbf{x x}}^{\dagger}$ and $U_{T_{x}, k}$, and matrix products $E_{\mathbf{y x}} E_{\mathbf{x x}}^{\dagger}, T_{x}$, and $A_{\mathrm{GB}} C_{\mathrm{GB}}$. The GKTL computational load ( $\left.L_{\mathrm{GKLT}}\right)$ consists of computation of matrix $E_{\mathbf{x x}}^{1 / 2^{\dagger}}$, matrix
TABLE I

ESTIMATES OF FLOP NUMBERS

\begin{tabular}{||c||c||c||}
\hline \multicolumn{1}{||c||}{} & \multicolumn{2}{c||}{ Flops } \\
\hline Required & Golub-Relisch SVD & $R$-SVD \\
\hline$U_{T_{x}}$ & $12 m^{3}$ & $13 m^{3}$ \\
\hline \hline SVD of $\Gamma E_{\mathbf{y x}}\left(E_{\mathbf{x x}}^{1 / 2}\right)^{\dagger}$ & $21 m^{3}$ & $26 m^{3}$ \\
\hline
\end{tabular}

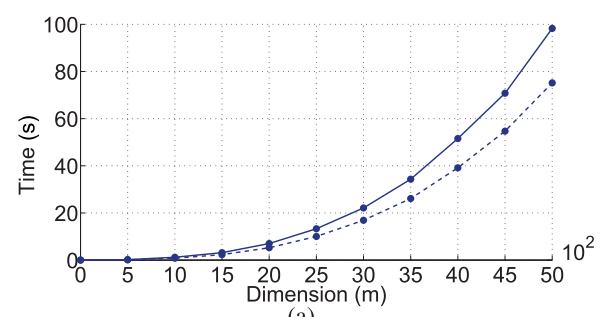

(a)

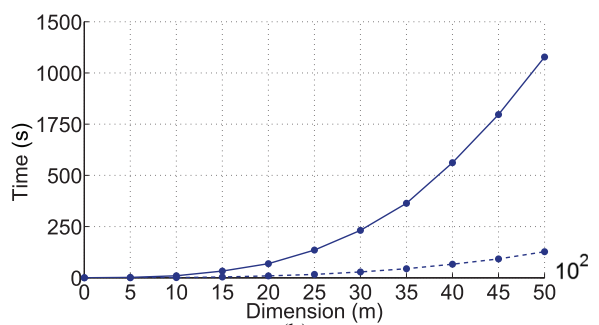

(b)

Fig. 1. Time versus matrix dimension $m$ used to execute the GBT1 (dashed line) and GKLT (solid line), for (a) $s=2 m$ and (b) $s=m / 2$.

product $\Gamma E_{\mathbf{y} \mathbf{x}} E_{\mathbf{x x}}^{1 / 2^{\dagger}}$, the SVD of $\Gamma E_{\mathbf{y x}} E_{\mathbf{x x}}^{1 / 2^{\dagger}}$, and matrix product $\Gamma^{\dagger}\left[\Gamma E_{\mathbf{y x}} E_{\mathbf{x x}}^{1 / 2^{\dagger}}\right]_{k} E_{\mathbf{x x}}^{1 / 2^{\dagger}}$. Matrix product of $m \times n$ and $n \times p$ requires approximately $2 m n p$ flops, for large $n$. On this basis, for $m=n, \Gamma=I, S=\mathbb{O}_{m, m}, R=\mathbb{O}_{n, n}$, and $U_{T_{x}}$ and the SVD of $E_{\mathbf{y x}} E_{\mathbf{x x}}^{1 / 2^{\dagger}}$ evaluated by the $R$-SVD

$$
L_{\mathrm{GBT} 1}=35 m^{3}+2 m^{2} k \quad \text { and } \quad L_{\mathrm{GKLT}}=52 m^{3} .
$$

For $U_{T_{x}}$ and the SVD of $E_{\mathbf{y x}} E_{\mathbf{x x}}^{1 / 2^{\dagger}}$ evaluated by the GolubRelisch SVD

$$
L_{\mathrm{GBT} 1}=34 m^{3}+2 m^{2} k \quad \text { and } \quad L_{\mathrm{GKLT}}=47 m^{3} .
$$

Of course, $L_{\mathrm{GBT} 1}$ and $L_{\mathrm{GKLT}}$ are evaluated approximately. A difference between $L_{\mathrm{GBT} 1}$ and $L_{\mathrm{GKLT}}$ increases if the covariance matrices are singular. Example (2) illustrates it.

Example 2: We simulate $\mathbf{y}$ and $\mathbf{x}$ by matrices $Y \in \mathbb{R}^{m \times s}$ and $X \in \mathbb{R}^{m \times s}$ as in Example (1) but with different $m, s$. The diagrams in Fig. 1(a) and (b) represent time versus matrix dimension $m$ used to execute the GBT1 and GKLT, for $c=m / 4$. For $s=m / 2$, the covariance matrices are singular. Fig. 1 illustrates the GBT1 advantage considered above, i.e., that the GBT1 is faster than the GKLT.

\section{Generalized Second-Order BT (GBT2)}

Now, we consider the generalized second-order BT (GBT2) in the form $F(\mathbf{x})=D C_{1} \mathbf{x}+D C_{2} \mathbf{v}$ that contains three matrices to optimize, $D, C_{1}$, and $C_{2}$, i.e., one matrix more than the GBT1. Here, $\mathbf{v}$ is an "auxiliary" signal used to further optimize the transform. We show that, because of the increase 
in a number of parameters to optimize, the GBT2 may provide better accuracy than that of the GBT1.

A. Determination of $D, C_{1}$, and $C_{2}$

Optimal $D \in \mathbb{R}^{m \times k}, C_{1} \in \mathbb{R}^{k \times n}$, and $C_{2} \in \mathbb{R}^{k \times n}$ solve

$$
\min _{D, C_{1}, C_{2}} \mathbb{E}\left[\left\|\Gamma\left(\mathbf{y}-D\left[C_{1} \mathbf{x}+C_{2} \mathbf{v}\right]\right)\right\|_{2}^{2}\right] .
$$

To find $D, C_{1}, C_{2}$, we denote $\mathbf{z}=\left[\begin{array}{l}\mathbf{x} \\ \mathbf{v}\end{array}\right], \quad N_{L}=I+(I-$ $\left.P_{\Gamma, R}\right) S$, and $N_{R}=I+K\left(I-P_{E_{\mathbf{z z}}^{1 / 2}, L}\right)$, where $S$ and $K$ are arbitrary matrices. Matrix $T_{z}$ and its SVD are defined similar to $T_{x}$ and the SVD of $T_{x}$, respectively, in Section II. Here, $\mathbf{v}$ is still arbitrary.

Theorem 2: Optimal $D=D^{(1)}$, and $C_{1}=C_{1}^{(1)}$ and $C_{2}=$ $C_{2}^{(1)}$ are given by

$$
D^{(1)}=N_{L} \Gamma^{\dagger} U_{T_{z}, k},\left[C_{1}^{(1)}, C_{2}^{(1)}\right]=U_{T_{z}, k}^{T} \Gamma E_{\mathbf{y z}} E_{\mathbf{z z}}^{\dagger} N_{R} .
$$

For the fixed compression ratio $c$, the associated error is

$$
\begin{aligned}
\varepsilon_{\mathrm{GBT} 2}(\mathbf{v}) & =\min _{D, C_{1}, C_{2}} \mathbb{E}\left[\left\|\Gamma\left(\mathbf{y}-D\left[C_{1} \mathbf{x}+C_{2} \mathbf{v}\right]\right)\right\|_{2}^{2}\right] \\
& =\left\|\left(\Gamma E_{\mathbf{y y}}\right)^{1 / 2}\right\|^{2}-\alpha_{2}(\mathbf{v})
\end{aligned}
$$

where $\alpha_{2}(\mathbf{v})=\sum_{j=1}^{r_{z}} \sigma_{j}\left(T_{z}\right), r_{z}=k$ if $k \leq \operatorname{rank}\left(T_{z}\right)$ and $r_{z}=\operatorname{rank}\left(T_{z}\right)$ if $k>\operatorname{rank}\left(T_{z}\right)$.

Proof: The proof is similar to the proof of Theorem 1 subject to the appropriate changes in notation.

\section{B. Determination of $\mathbf{v}$}

1) First Method: The practical approach is to replace the cost in (15) with the sample version, $J\left(D, C_{1}, C_{2}, Y, V\right):=$ $\left\|\Gamma\left(Y-D\left[C_{1} X+C_{2} V\right]\right)\right\|^{2}=\|G-H V\|^{2}$,

where $Y \in \mathbb{R}^{m \times s}, X \in \mathbb{R}^{n \times s}$ are samples of $\mathbf{y}, \mathbf{x}$, respectively, $V \in \mathbb{R}^{n \times s}, G=\Gamma\left(Y-D C_{1} X\right)$ and $H=\Gamma D C_{2}$. In this setting, we determine optimal $V$ iteratively as follows.

First, for an arbitrary $V$, determine optimal $D^{(1)}, C_{1}^{(1)}$, $C_{2}^{(1)}$ such that $J\left(D^{(1)}, C_{1}^{(1)}, C_{2}^{(1)}, Y, V\right)=\min _{D, C_{1}, C_{2}} J(D$, $\left.C_{1}, C_{2}, Y, V\right)$. Then, $D^{(1)}, C_{1}^{(1)}, C_{2}^{(1)}$ are given as in (16) where $E_{\mathbf{y z}}$ is replaced with $s^{-1} Y Z^{T}$ where $Z=\left[X^{T}, V^{T}\right]^{T}$.

Write $Y^{(1)}=\Gamma^{\dagger} \Gamma D^{(1)}\left[C_{1}^{(1)} X+C_{2}^{(1)} V\right]$. Second, find $D^{(2)}, C_{1}^{(2)}, C_{2}^{(2)}$ such that $J\left(D^{(2)}, C_{1}^{(2)}, C_{2}^{(2)}, Y^{(1)}, V\right)=$ $\min _{D, C_{1}, C_{2}} J\left(D, C_{1}, C_{2}, Y^{(1)}, V\right)$. Find the minimalnorm $V^{(1)}$ such that $J\left(D^{(2)}, C_{1}^{(2)}, C_{2}^{(2)}, Y^{(1)}, V^{(1)}\right)=$ $\min _{V} J\left(D^{(2)}, C_{1}^{(2)}, C_{2}^{(2)}, Y^{(1)}, V\right)$. Then,

$$
V^{(1)}=H^{(2)^{\dagger}} \Gamma\left(Y^{(1)}-D^{(2)} C_{1}^{(2)} X\right),
$$

where $H^{(2)}=\Gamma D^{(2)} C_{2}^{(2)}$. Then the procedure is repeated with $V^{(1)}$ instead of $V$ until the tolerance for the estimate of $Y$ is achieved.

Convergence will be elaborated and reported elsewhere in the near future.

2) Second Method: In particular, $\mathbf{v}$ can be chosen as $\mathbf{v}=$ $\mathbf{x}^{2}$, where $\mathbf{x}^{2}$ is given by $\mathbf{x}^{2}(\omega)=\left[\mathbf{x}_{1}^{2}(\omega), \ldots, \mathbf{x}_{n}^{2}(\omega)\right]^{T}$ and $\mathbf{x}_{j}^{2}(\omega)=\left[\mathbf{x}_{j}(\omega)\right]^{2}$. Although this choice of $\mathbf{v}$ is simple, it is not optimal.

Furthermore, the error representations in (5) and (17) imply $\varepsilon_{\mathrm{GBT} 1}-\varepsilon_{\mathrm{GBT} 2}(\mathbf{v})=\alpha_{2}(\mathbf{v})-\alpha_{1}$. Therefore, if $\alpha_{1}<\alpha_{2}(\mathbf{v})$

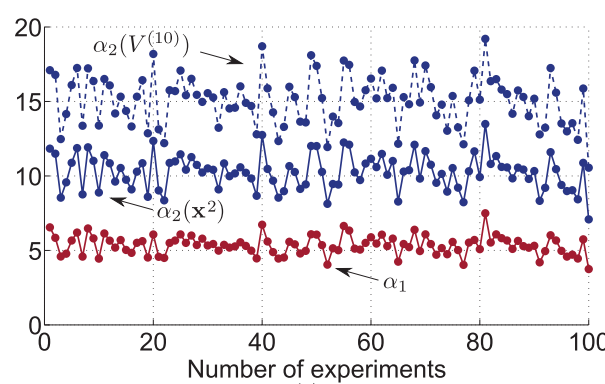

(a)

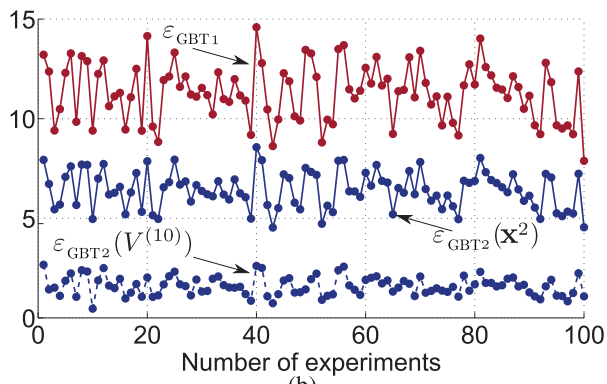

(b)

Fig. 2. Example 4: Illustrations to conditions $\alpha_{1}<\alpha_{2}\left(\mathbf{x}^{2}\right), \quad \alpha_{1}<$ $\alpha_{2}\left(V^{(10)}\right)$ (a) and MSEs (b).

then, for the same compression ratio $c$, the error associated with the GBT2 is less than that of the GBT1, i.e., $\varepsilon_{\mathrm{GBT} 2}(\mathbf{v})<\varepsilon_{\mathrm{GBT} 1}$. The condition $\alpha_{1}<\alpha_{2}(\mathbf{v})$ can be used in testing experiments as those in Examples (3) and (4) below.

\section{Models of Compressor and Decompressor by GBT2}

Compressed signal is represented by $\mathbf{u}=C_{1} \mathbf{x}+C_{2} \mathbf{v}$. Compressor and decompressor are represented by $C_{1}$ and $C_{2}$, and $D$, respectively.

Example 3: Let $\boldsymbol{x}=\boldsymbol{y}+\sigma^{2} \boldsymbol{\xi}$ where $\boldsymbol{y} \in L^{2}\left(\Omega, \mathbb{R}^{100}\right)$ is a uniformly distributed random vector, $\sigma \in \mathbb{R}, \mathbf{v}=\mathbf{x}^{2}$ and $\boldsymbol{\xi} \in L^{2}\left(\Omega, \mathbb{R}^{100}\right)$ is white noise. Vectors $\boldsymbol{x}, \boldsymbol{y}$, and $\boldsymbol{\xi}$ are assumed to be independent. Therefore, $E_{\mathbf{y y}}=$ $\left\{\eta_{i j}\right\}_{i, j=1}^{100}, \quad \eta_{i j}=\left\{\begin{array}{ll}1 / 3, & i=j, \\ 1 / 4, & i \neq j,\end{array} E_{\mathbf{y x}}=E_{\mathbf{y y}}, \quad E_{\boldsymbol{\xi} \xi}=\sigma^{2} I\right.$, $E_{\mathbf{x x}}=E_{\mathbf{y y}}+E_{\xi \xi}, E_{\mathbf{y} \mathbf{x}^{2}}=\left\{\lambda_{i j}\right\}_{i, j=1}^{100}, \lambda_{i j}= \begin{cases}1 / 4, & i=j \\ 1 / 6, & i \neq j,\end{cases}$ $E_{\mathbf{y z}}=\left[E_{\mathbf{y y}} E_{\mathbf{y x}^{2}}\right], \quad$ where $\quad \mathbf{z}=\left[\mathbf{x}^{T}(\mathbf{v})^{T}\right]^{T}, \quad E_{\mathbf{x}^{2} \mathbf{x}^{2}}=$ $\left\{\mu_{i j}\right\}_{i, j=1}^{100}, \quad \mu_{i j}\left\{\begin{array}{ll}1 / 5, & i=j \\ 1 / 9, & i \neq j,\end{array} E_{\xi^{2} \xi^{2}}=2 \sigma^{4} I, \quad\right.$ and $\quad E_{\mathbf{z z}}=$ $\left[\begin{array}{cc}E_{\mathbf{x x}} & E_{\mathbf{y y}} \\ E_{\mathbf{y y}} & E_{\mathbf{x}^{2} \mathbf{x}^{2}}+E_{\xi^{2} \xi^{2}}\end{array}\right]$. Let, e.g., $k=50$ and $\sigma=0.4$. Then, $\alpha_{1}=27.7, \alpha_{2}=30.0$, and $\varepsilon_{\mathrm{GBT} 1}=7.01, \varepsilon_{\mathrm{GBT} 2}=5.8$.

Example 4: Let $\boldsymbol{y}$ be a uniformly distributed random vector, $\mathbf{x}=s_{1} \mathbf{y}+s_{2} \boldsymbol{\delta}, s_{1}, s_{2} \in[0.1,10]$ and $\boldsymbol{\delta}$ is a Gaussian random vector with mean zero and variance one. The covariance matrices are formed from samples $Y \in \mathbb{R}^{50 \times 150}$ and $X \in \mathbb{R}^{50 \times 150}$. In each test, $Y, X$, and diagonal $\Gamma$ with entries within $(0,1)$ are chosen randomly. In Fig. 2(a), for $s_{1}=0.5$ and $s_{2}=8$, conditions $\alpha_{1}<\alpha_{2}\left(\mathbf{x}^{2}\right), \alpha_{1}<\alpha_{2}\left(V^{(10)}\right)$ are illustrated where values of $\alpha_{1}$ and $\alpha_{2}$ are represented versus experiment numbers. In Fig. 2(b), for the same $s_{1}$ and $s_{2}$, values of the errors are given. In all 100 experiments, the GBT2 with optimized $V$ given by $V^{(10)}$ provides better associated accuracy. In fact, Fig. 2 represents typical diagrams of the experiments we made for different values of $s_{1}, s_{2} \in[0.1,10]$. 


\section{REFERENCES}

[1] D. R. Brillinger, Time Series: Data Analysis and Theory. San Francisco, CA, USA: Holden Day, 2001

[2] Y. Hua, M. Nikpour, and P. Stoica, "Optimal reduced-rank estimation and filtering," IEEE Trans. Signal Process., vol. 49, no. 3, pp. 457-469, Mar. 2001.

[3] I. D. Schizas, G. B. Giannakis, and Z.-Q. Luo, "Distributed estimation using reduced-dimensionality sensor observations," IEEE Trans. Signal Process., vol. 55, no. 8, pp. 4284-4299, Aug. 2007.

[4] T. Piotrowski IsaoYamada, "Convex formulation of the stochastic MVPURE estimator and its relation to the reduced rank Wiener filter," in Proc. Int. Conf. Signals Electron. Syst. (ICSES'08), 2009, pp. 3081-30 843.

[5] T. Piotrowski and I. Yamada, "Why the stochastic MV-PURE estimator excels in highly noisy situations?" in Proc. IEEE Int. Conf. Acoust., Speech, Signal Process. (ICASSP'09), 2009, pp. 3081-30 843.

[6] T. Piotrowski and I. Yamada, "Stochastic MV-PURE estimator-Robust reduced-rank estimator for stochastic linear model," IEEE Trans. Signal Process., vol. 57, no. 4, pp. 1293-1303, Apr. 2009.

[7] T. Piotrowski and I. Yamada, "Performance of the stochastic MV-PURE estimator in highly noisy settings," J. Franklin Insit., vol. 351, pp. 33393350, 2014.

[8] A. Torokhti and P. Howlett, Computational Methods for Modelling of Nonlinear Systems. New York, NY, USA: Elsevier, 2007.

[9] L. L. Scharf, "The SVD and reduced rank signal processing," Signal Process., vol. 25, no. 2, pp. 113-133, 1991.
[10] Y. Hua and W. Liu, "Generalized Karhunen-Loeve transform," IEEE Signal Process. Lett., vol. 5, no. 6, pp. 141-142, Jun. 1998.

[11] N. Srebro and T. Jaakkola, "Weighted low-rank approximations," in Proc. 20th Int. Conf. Mach. Learn. (ICML'03), Washington DC, USA, 2003, pp. $720-727$.

[12] D. G. Manolakis, V. K. Ingle, and S. M. Kogon, Statistical and Adaptive Signal Processing: Spectral Estimation, Signal Modeling, Adaptive Filtering, and Array Processing. Norwood, MA, USA: Artech House, 2005.

[13] P. Bühlmann and S. van de Geer, Statistics for High-Dimensional Data. New York, NY, USA: Springer, 2011.

[14] S. Friedland and A. Torokhti, "Generalized rank-constrained matrix approximations," SIAM J. Matrix Anal. Appl., vol. 29, no. 2, pp. 656-659, 2007.

[15] A. Torokhti and S. Friedland, "Towards theory of generic principal component analysis," J. Multivariate Anal., vol. 100, no. 4, pp. 661-669, 2009.

[16] O. Alter and G. H. Golub, "Singular value decomposition of genomescale mRNA lengths distribution reveals asymmetry in RNA gel electrophoresis band broadening," Proc. Natl. Acad. Sci. USA, vol. 103, no. 32, pp. 11 828-11 833, 2006.

[17] Z. Yang and G. Michailidis, "A non-negative matrix factorization method for detecting modules in heterogeneous omics multi-modal data," Bioinformatics, vol. 103, no. 32, pp. 1-8, 2015.

[18] G. Golub and C. F. V. Loan, Matrix Computations. Baltimore, MD, USA The Johns Hopkins Univ. Press, 1996. 